\title{
Universiteit
}

Leiden

The Netherlands

\section{Experimental observation of wave chaos in a conventional optical} resonator

Dingjan, J.; Altewischer, E.; Exter, M.P. van; Woerdman, J.P.

\section{Citation}

Dingjan, J., Altewischer, E., Exter, M. P. van, \& Woerdman, J. P. (2002). Experimental observation of wave chaos in a conventional optical resonator. Physical Review Letters, 88(6), 064101. doi:10.1103/PhysRevLett.88.064101

Version: $\quad$ Not Applicable (or Unknown)

License: $\quad$ Leiden University Non-exclusive license

Downloaded from: https://hdl.handle.net/1887/64335

Note: To cite this publication please use the final published version (if applicable). 


\title{
Experimental Observation of Wave Chaos in a Conventional Optical Resonator
}

\author{
J. Dingjan,* E. Altewischer, M. P. van Exter, and J.P. Woerdman \\ Huygens Laboratory, Leiden University, P.O. Box 9504, 2300 RA Leiden, The Netherlands
}

(Received 21 June 2001; published 28 January 2002)

\begin{abstract}
We report on an experimental observation of optical wave chaos in a resonator consisting of three standard, high-reflectivity mirrors. The nonseparability of the wave equation necessary for chaos is introduced by violating the paraxial approximation. Until recently progress in optical wave chaos was hampered by the inherent difficulty in realizing suitable microscopic systems; now this novel, macroscopic approach offers complete and easy control and allows unprecedented study of optical wave chaos.

DOI: 10.1103/PhysRevLett.88.064101

PACS numbers: 05.45.Mt, 42.60.Da, 42.65.Sf
\end{abstract}

Optical wave chaos is a topical field with highlights such as localization of light and random laser action [1-8]. The material systems used so far are microscopic in nature; these systems either have disorder present in the bulk (powders [2,5] and suspensions $[1,4]$ ) or they show chaotic behavior due to the nonseparability of the boundary conditions (oval-shaped dielectric microresonators $[7,8]$ ). These microscopic systems are generally difficult to fabricate and reproduce and this has limited the experimental progress in this field.

In contrast, wave-chaos experiments in the microwave domain allow much better control; for instance, the use of closed, stadium-type microwave resonators has led to spectacular progress [9]. Extension of this degree of control into the optical domain would be fundamentally important since optical waves may display quantum aspects (spontaneous emission and lasing), contrary to microwaves.

In this Letter, we propose and demonstrate an optical system that allows easy fabrication and superb control. We have observed optical wave chaos in a macroscopic, geometrically stable open resonator, formed by three standard high-reflectivity mirrors, where nonseparability of the wave equation is realized by violating the paraxial approximation (Fig. 1). In historical perspective, we employ the key idea to construct a laser, as introduced by Schawlow and Townes [10], namely, the use of an open instead of a closed resonator, but now in the context of wave chaos.

The violation of paraxiality destroys the separability of the intracavity light field into the usual longitudinal and transverse Hermite-Gaussian modes. In lowest order, nonparaxiality can be parametrized in terms of the five Seidel aberrations which can be found in any standard optics textbook [11]: coma, astigmatism, spherical aberration, field curvature, and distortion. Because of these aberrations the mode indices labeling the Hermite-Gaussian modes lose their meaning, especially for higher order modes. In such a case we can no longer find a complete set of good quantum numbers (or constants of motion). Depending on the symmetry of the aberrations and their relative orientations, one or both transverse mode indices are invalidated.

To achieve the required amount of nonparaxiality in a two-mirror cavity, its numerical aperture (NA) must be- come inconveniently large. Instead, we use a three-mirror folded linear cavity, formed by two high-reflectivity end mirrors, which can be either flat or curved, and a highreflectivity curved folding mirror (see Fig. 1). This curved folding mirror introduces very large aberrations [11], allowing us to realize an effectively strong nonparaxiality, and thereby nonseparability, with only a very modest NA, so that standard high-reflectivity mirrors can be used. The question whether this is sufficient to realize chaotic dynamics seems very difficult to answer theoretically, since calculations of very-high-order transverse modes in nonparaxial 3D open resonators are not yet within reach (contrary to the case of closed resonators). Therefore, we have addressed this question with experiments.

The most striking effects of wave chaos can be found in the spectral properties of chaotic systems. As a diagnostic tool, the nearest-neighbor level spacing statistics has received the most attention, as this will show strong level repulsion for chaotic systems, contrary to the regular case. For this method, and related techniques, it is essential that every eigenfrequency in an interval can be resolved, since missing, or spurious, peaks will quickly obscure the results. As we explain below, the eigenfrequency spectra for our system show many overlapping peaks, making it impossible to extract every eigenfrequency. As a result, direct spectral statistics are not feasible.

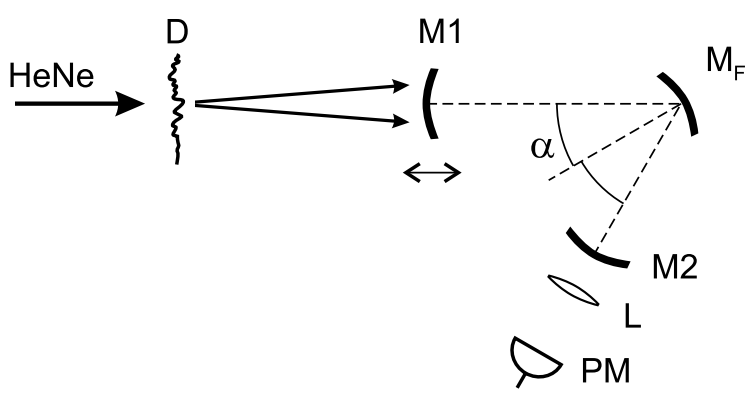

FIG. 1. Experimental setup. HeNe: helium-neon laser beam, D: diffusor, $\mathrm{M} 1, \mathrm{M} 2$ : resonator end mirrors, $\mathrm{M}_{\mathrm{F}}$ : folding mirror, L: lens, and PM: photomultiplier. The figure shows the aberrated resonator, which has a concave folding mirror $\mathrm{M}_{\mathrm{F}}$. The use of a flat folding mirror leads to a nonaberrated resonator. 
Fortunately, a diagnostic method dealing with such "dirty," potentially chaotic, spectra has been developed in the context of highly excited molecules $[12,13]$. We follow in particular the treatment of Wilkie and Brumer [14], which is in effect a time-domain approach. The key quantity is the wave packet survival probability function $P(t)$, which describes the survival probability after a mixing time $t$ of an initial wave packet. $P(t)$ can either be found from the normalized wave function $\psi(t)$ or can be determined from the Fourier transform of the normalized eigenfrequency spectrum $S(\omega)$,

$$
\begin{aligned}
& P(t) \equiv|\langle\psi(0) \mid \psi(t)\rangle|^{2}=\left|\int S(\omega) e^{-i \omega t} d \omega\right|^{2}, \\
& P(0)=1 .
\end{aligned}
$$

Note that $P(t)$ is the Fourier transform of the spectral autocorrelation function. Methods using $P(t)$ do not require peak finding and are, therefore, in the case of imperfect spectra with missing, spurious, or overlapping peaks, superior to frequency domain methods [12].

After performing a double averaging, over both the initial conditions and different realizations of the system, we obtain $\langle\langle P(t)\rangle\rangle$. Through Fourier relations, short-range spectral correlations, such as the nearest-neighbor distribution, affect the long time limit of $\langle\langle P(t)\rangle\rangle$, while the short time limit of $\langle\langle P(t)\rangle\rangle$ is determined by long-range spectral correlations, e.g., the variance of the number of lines in a long interval [9]. This short time limit is expected to contain the useful information when dealing with limited spectral resolution in experiments. Wilkie and Brumer [14] have shown theoretically that for a regular system $\langle\langle P(t)\rangle\rangle \geq\langle\langle P(\infty)\rangle\rangle$ for all $t \geq 0$, while for a chaotic system $\langle\langle P(t)\rangle\rangle\langle\langle\langle P(\infty)\rangle\rangle$ for at least some values of $t$. In other words, $\langle\langle P(t)\rangle\rangle$ must fall below its long time asymptote in the case of chaotic dynamics, while it cannot do so in the case of regular dynamics. Hence, this time-domain method allows an unambiguous identification of the nature of the dynamics. In essence, the lack of instrumental resolution, which makes direct extraction of spectral statistics from a single spectrum impossible, is compensated for by averaging over an ensemble of many realizations.

This method applies equally to closed and open Hamiltonian systems; in the latter case one must take out the trivial decay factor in $\langle\langle P(t)\rangle\rangle$, caused by the dissipation, leading to $\langle\langle P(t)\rangle\rangle^{\prime}$ instead of $\langle\langle P(t)\rangle\rangle$. One then finds as asymptotic behavior $\langle\langle P(\infty)\rangle\rangle^{\prime} \sim 2 /(N+1)$, where $N$ is the number of lines in the spectral interval under consideration. When applied to an optical resonator, $S(\omega)$ is the intensity transmission spectrum, $\psi(t)$ is a short-hand notation for the spatiotemporal field pattern in the resonator, and $N$ is the number of excited transverse modes.

Our resonator consisted of three high-reflectivity concave mirrors with a diameter of $25 \mathrm{~mm}$ and a radius of curvature of $1 \mathrm{~m}$ (Fig. 1). The half folding angle of the resonator was chosen as $\alpha=45^{\circ}$. The length of the arms was $\sim 10 \mathrm{~cm}$ and $\sim 19 \mathrm{~cm}$; this yields roughly NA $\sim 0.04$. These values correspond to the paraxially stable regime of the folded resonator. A total length $L \sim 29 \mathrm{~cm}$ leads to a free spectral range (FSR) $\nu_{\mathrm{FSR}} \equiv t_{\text {round-trip }}^{-1}=c / 2 L \sim$ $0.5 \mathrm{GHz}$, where $t_{\text {round-trip }}$ is the cavity round-trip time.

We determine the eigenfrequency spectrum of the resonator by injecting a monochromatic HeNe laser beam $(\lambda=$ $633 \mathrm{~nm}$ ) and by measuring the transmitted intensity while scanning the cavity length $L$ over a few wavelengths. Before entering the cavity, the HeNe beam (with a diameter of $\sim 0.8 \mathrm{~mm}$ ) passes through a weak diffusor; this diffusor produces a speckled input field and thus allows an appreciable spatial overlap with a large number of transverse modes. The number of excited modes, $N$, can be adjusted by varying the distance between the diffusor and the cavity and by choosing a diffusor with a different scattering strength. We may estimate the number of excited modes from the typical radius $a$ of the light spot on the mirrors, $N \sim a^{4} / \lambda^{2} L^{2} \sim 10^{4}[15]$.

A key parameter is the finesse $\mathcal{F}$ of the resonator, defined as $\nu_{\mathrm{FSR}} / \Delta \nu$, where $\Delta \nu$ is the width of an individual mode. If we remove the diffusor and paraxially inject a laser beam into the resonator, thereby exciting only a few, low-order, transverse modes, we obtain $\mathcal{F}=$ $1.8 \times 10^{3}\left(Q \equiv \nu / \Delta \nu=1.6 \times 10^{9}\right)$. This value is consistent with the independently measured reflectivities of the three mirrors. Since $N>\mathcal{F}$ the transmission spectrum will be largely "filled" due to the overlap of peaks, so that peak-finding methods cannot be used.

Experimentally, this filling is illustrated by the transmission spectrum shown in Fig. 2a. An important observation is that all peaks that, through chance, can be individually analyzed, keep their Lorentzian shape, and show the same finesse as for a resonator in which only a few modes are excited. This indicates that, in the presence of aberrations,

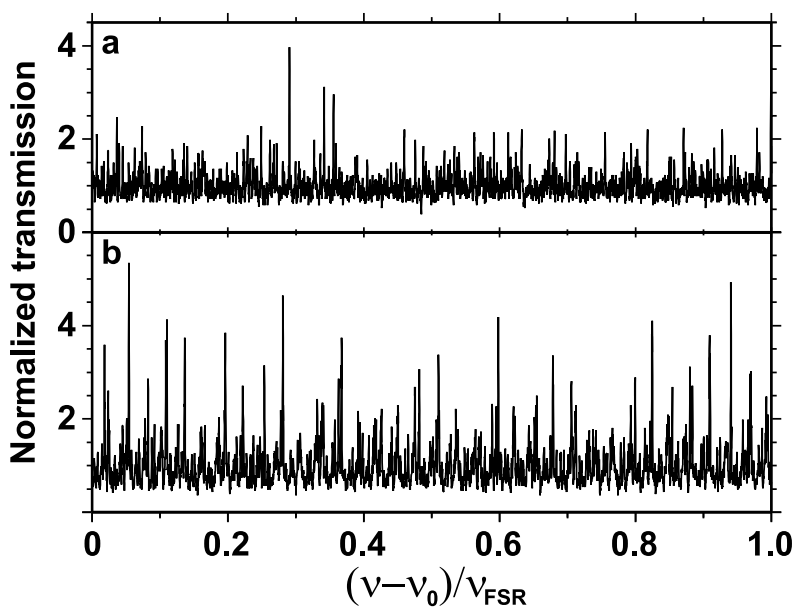

FIG. 2. Transmission spectra, normalized to the transmission averaged over one free spectral range, for (a) an aberrated resonator, using a concave folding mirror (chaotic dynamics), and (b) a nonaberrated resonator, using a flat folding mirror (regular dynamics). 
the mode manifold of the (paraxially stable) resonator remains largely decoupled from the free space modes.

The required averaging over initial conditions can be achieved by averaging transmission spectra of the resonator for various transverse positions of the diffusor. Averaging over different realizations of the system is obtained by varying the length of one of the arms of the resonator over a relatively large range $\Delta L$, typically $\sim 1 \mathrm{~mm}$ (i.e., much larger than the wavelength of the HeNe light). This interval is sufficiently large to yield many different intracavity field realizations since it corresponds to a change of the Fresnel number $N_{F} \equiv a^{2} / \lambda L$ by an amount of order unity [16].

We have chosen ten transverse positions of the diffusor, combined with 40 resonator arm lengths, yielding a total of 400 transmission spectra. After normalizing with respect to the area under the spectrum and calculating the squared modulus of the Fourier transform, all results were averaged. In Fig. 3, we have plotted as the black curve the thus obtained $\langle\langle P(t)\rangle\rangle^{\prime}$, i.e., $\langle\langle P(t)\rangle\rangle$ corrected for the finite cavity finesse, using the independently obtained value for $\mathcal{F}$. For small $t / t_{\text {round-trip }}$, we observe a well-developed dip where $\langle\langle P(t)\rangle\rangle^{\prime}\left\langle\langle\langle P(\infty)\rangle\rangle^{\prime}\right.$; this convincingly proves the chaotic nature of the dynamics in our aberrated resonator.

Chaotic spectra as shown in Fig. 2a could be obtained only around certain settings of the arm lengths of the resonator. Other settings led to quasiperiodic spectra. This is quantified by Fig. 4, where we plot the magnitude of the principal-frequency Fourier component as a function of the cavity length offset. We have preliminary evidence that these quasiperiodic spectra are associated with scars [17]; this will be reported in a future publication. In the present Letter we restrict ourselves to cavity lengths where these quasiperiodic spectra do not obscure chaoticity. Around these lengths there are sufficiently large intervals $(\sim 1 \mathrm{~mm})$ to allow the many realizations that we need for our diagnostics. We have checked that "nonquasiperiodic" intervals other than the one indicated in Fig. 4 yield results similar to the black curve in Fig. 3.

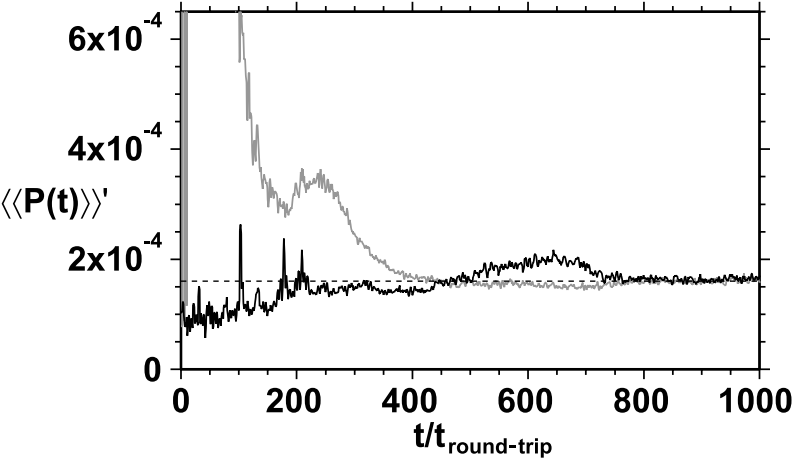

FIG. 3. Wave packet survival probability function, averaged over initial conditions and an ensemble of realizations, corrected for the finite finesse. Black curve: aberrated resonator (chaotic dynamics). Grey curve: nonaberrated resonator (regular dynamics). Dashed line: long time asymptote.
The shape and depth of the dip in the $\langle\langle P(t)\rangle\rangle^{\prime}$ curve are roughly as observed for the nuclear data ensemble (NDE), where fully developed chaos has been independently established [18]; this suggests that also in our system the chaos is reasonably well developed. Furthermore, we see that, for $t / t_{\text {round-trip }}>800,\langle\langle P(t)\rangle\rangle^{\prime}$ reaches its asymptotic value of $2 /(N+1) \approx 1.6 \times 10^{-4}$, from which we deduce $N \approx 1.25 \times 10^{4}$. This value agrees with our rough a priori estimate.

Our results are even more convincing when we contrast them with $\langle\langle P(t)\rangle\rangle^{\prime}$ obtained from 600 transmission spectra of a comparable regular resonator. For this, we have replaced the curved folding mirror with a planar one, again with high reflectivity. Since it is well known that a planar mirror does not induce aberrations, this turns the cavity into a trivially folded, effectively two-mirror resonator. In Figs. 2a and $2 \mathrm{~b}$ we compare the transmission spectra for the two cases. Both spectra are "grassy," but very different, as are the $\langle\langle P(t)\rangle\rangle^{\prime}$ curves plotted in Fig. 3. For the effective two-mirror resonator we observe that, within the experimental uncertainty, $\left.\langle\langle P(t)\rangle\rangle^{\prime}\right\rangle\langle\langle P(\infty)\rangle\rangle^{\prime}$ for all $t$, thus confirming the regular nature of the dynamics. Furthermore, the largest deviation between $\langle\langle P(t)\rangle\rangle^{\prime}$ obtained for an aberrated and a nonaberrated resonator occurs for small $t$, as expected.

It is gratifying to see that the nonparaxiality due to the curved folding mirror is strong enough to produce chaos. It would be very interesting to see how the strength of this chaos depends on the cavity configuration. For instance, we may study the onset of chaos as we increase the amount of nonseparability from zero; this can be done continuously, by varying either the NA or the folding angle $\alpha$, or both. Also, it seems possible to increase the number of modes to $10^{6}$ (in the setup of Fig. 1 this corresponds to $\mathrm{NA} \approx 0.12$ ) or more, exceeding what can be achieved in microwave billiards [9]. Furthermore, we expect that

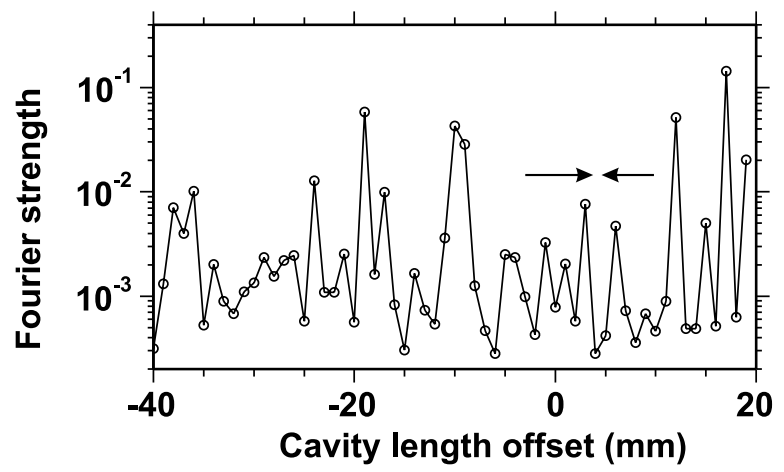

FIG. 4. Magnitude of the principal-frequency Fourier component of the spectrum of the aberrated resonator of Fig. 1. This magnitude is plotted as a function of the length offset of the variable arm of the cavity. The curve is meant to guide the eye; its shape is, as yet, unexplained. Note that the vertical scale is logarithmic. Indicated is the $1-\mathrm{mm}$ interval with relatively weak quasiperiodicity which has been used to obtain the black curve of Fig. 3. 
a nonplanar standing wave or ring resonator, again with curved folding mirrors, will produce even stronger chaos due to its lower symmetry.

The perfect spatial, temporal, and polarization control offered by a standard optical resonator offers exciting perspectives for study of optical phenomena based on wave chaos. A prime example is localization of light, a field where many open questions remain $[3,19]$. Weak localization or coherent backscattering [1] can be studied by measuring the angle-resolved backscatter of the injected $\mathrm{HeNe}$ beam from the resonator. We expect that a phase transition to strong (or Anderson) localization [2] may occur as soon as the global disorder due to the Seidel aberrations has sufficient strength. Strong localization would imply that a wide light beam, injected into the resonator, splits up, due to destructive interference of transverse ray components, into a number of diffraction limited filaments with transverse extents of $\sim \lambda /$ NA. This can be studied both spatially, using a CCD camera to record the intensity pattern on one of the mirrors, and spectrally, through the disappearance of level repulsion.

All this can be easily carried over from passive to active systems, since it is simple to insert a gain medium into our resonator. We may thus, for instance, build a (continuously pumped) random laser; this will enable a detailed study of the intriguing nature of threshold transition and quantum noise that have been predicted for random lasers $[4,20]$. Breaking time-reversal symmetry is expected to drastically affect the properties of a random laser [21]; this, too, can easily be achieved, namely, by inserting a Faraday polarization rotator in a nonparaxial ring resonator.

In conclusion, we have realized optical wave chaos in an open resonator consisting of three standard, highreflectivity mirrors. The presence or absence of chaotic behavior is determined only by the geometry of the resonator: using a curved folding mirror, which introduces strong aberrations, leads to chaotic dynamics, while a flat folding mirror, which does not introduce aberrations, leads to regular dynamics. The deepest reason for this is that geometrical optics is essentially nonlinear as soon as one goes beyond the paraxial approximation [22].

This work was supported by the "Stichting voor Fundamenteel Onderzoek der Materie" (FOM).
*Email address: dingjan@molphys.leidenuniv.nl

[1] M. P. van Albada and A. Lagendijk, Phys. Rev. Lett. 55, 2692 (1985).

[2] D. S. Wiersma, P. Bartolini, A. Lagendijk, and R. Righini, Nature (London) 390, 671 (1997).

[3] B. A. van Tiggelen, in Diffuse Waves in Complex Media, edited by J.-P. Fouque (Kluwer, Dordrecht, 1999), p. 1.

[4] G. van Soest, F. J. Poelwijk, R. Sprik, and A. Lagendijk, Phys. Rev. Lett. 86, 1522 (2001).

[5] H. Cao, Y. Ling, J. Y. Xu, C. Q. Cao, and P. Kumar, Phys. Rev. Lett. 86, 4524 (2001).

[6] G. Zacharakis, N. A. Papadogiannis, G. Filippidis, and T. G. Papazoglou, Opt. Lett. 25, 923 (2000).

[7] C. Gmachl et al., Science 280, 1556 (1998).

[8] S. Chang, R. K. Chang, A. D. Stone, and J. U. Nöckel, J. Opt. Soc. Am. B 17, 1828 (2000).

[9] H.-J. Stöckmann, Quantum Chaos: An Introduction (Cambridge University Press, Cambridge, 1999).

[10] A. L. Schawlow and C. H. Townes, Phys. Rev. 112, 1940 (1958).

[11] M. Born and E. Wolf, Principles of Optics (Cambridge University Press, Cambridge, 1999).

[12] L. Leviandier, M. Lombardi, R. Jost, and J. P. Pique, Phys. Rev. Lett. 56, 2449 (1986); J. P. Pique, Y. Chen, R. W. Field, and J. L. Kinsey, ibid. 58, 475 (1987).

[13] In the molecular case, chaos occurs for sufficiently large quantum numbers; in the case of a nonparaxial resonator, chaos occurs for sufficiently large transverse mode indices.

[14] J. Wilkie and P. Brumer, Phys. Rev. Lett. 67, 1185 (1991).

[15] We use a diffusor with a scattering half cone angle of $0.5^{\circ}$, placed $20 \mathrm{~cm}$ before the first mirror; the spots on the mirrors then have diameters of the order of $1 \mathrm{~cm}$.

[16] The transverse intracavity field is roughly a periodic function of $N_{F}$, with period 1 .

[17] E. J. Heller, Phys. Rev. Lett. 53, 1515 (1984).

[18] See, for example, Fig. 1d of Leviandier et al. [12].

[19] T. Prosen, in New Directions in Quantum Chaos, Proceedings of the International School of Physics "Enrico Fermi," Course CXLIII, edited by G. Casati, I. Guarneri, and U. Smilansky (IOS Press, Amsterdam, 2000), p. 473.

[20] C. W. J. Beenakker, Phys. Rev. Lett. 81, 1829 (1998); G. Hackenbroich, C. Viviescas, B. Elattari, and F. Haake, ibid. 86, 5262 (2001).

[21] F. Haake, Quantum Signatures of Chaos (Springer-Verlag, Berlin, 1991).

[22] See Eq. (15) on p. 119 of [11]. 